\title{
Effects of Paired and Unpaired Eye-Blink Conditioning on Purkinje Cell Morphology
} Brenda J. Anderson, ${ }^{1,5}$ Karen Relucio, ${ }^{2,6}$ Karl Haglund, ${ }^{2,7}$ Christy Logan, ${ }^{3}$ Barbara Knowlton, ${ }^{3,8}$ Judith Thompson, ${ }^{3}$ Joseph E. Steinmetz, ${ }^{4}$ Richard F. Thompson, ${ }^{3}$ and William T. Greenough ${ }^{2}$

${ }^{1}$ Department of Psychology

State University of New York-Stony Brook

Stony Brook, New York 11794 USA

${ }^{2}$ Beckman Institute

Center for Neurobiology of Learning and Memory

Departments of Psychology, Cell, and Structural Biology

and the Neuroscience Program

University of Illinois

Urbana, Illinois 61801 USA

${ }^{3}$ Neuroscience Program

University of Southern California

Los Angeles, California 90089 USA

${ }^{4}$ Program in Neuroscience

Indiana University

Bloomington, Indiana 47401 USA

\begin{abstract}
This experiment addressed (1) the importance of conjunctive stimulus presentation for morphological plasticity of cerebellar Purkinje cells and inhibitory interneurons and (2) whether plasticity is restricted to the spiny branches of Purkinje cells, which receive parallel fiber input. These issues were investigated in naive rabbits and in rabbits that received paired or unpaired presentations of the conditioned stimulus (CS) and unconditioned stimulus (US). To direct CS input to the cerebellar cortex, pontine stimulation served as the CS. Air puffs to the cornea served as the US. Paired condition rabbits received pontine stimulation for 350 msec paired with a coterminating 100-msec
\end{abstract}

${ }^{5}$ Corresponding author.

Present addresses: ${ }^{6}$ Rush Medical College, Chicago, Illinois 60612 USA; ${ }^{7}$ Yale University School of Medicine, New Haven, Connecticut 06510 USA; ${ }^{8}$ Department of Psychology, University of California, Los Angeles, California 90095 USA. air puff. Unpaired condition rabbits received the same stimuli in a pseudorandom order at 1- to 32-sec intervals. Rabbits were trained for a mean of 12 days. Naive rabbits received no treatment. In Golgi-stained Purkinje neurons in lobule HVI, total dendritic length, main branch length, total spiny branch length, and number of spiny branch arbors were all greater in the naive group than in the paired and unpaired groups, which did not differ. No differences were found between the hemispheres ipsilateral and contralateral to the trained eye. The dendritic length and number of branches for inhibitory interneurons did not differ across groups. The Purkinje cell morphological changes detected with these methods do not appear to be uniquely related to the conjunctive activation of the CS and US in the paired condition.

\section{Introduction}

Both mathematical models of cerebellar learning and empirical data have suggested that conjunctive activation of parallel fibers and climbing

LEARNING \& MEMORY 6:128-137 @ 1999 by Cold Spring Harbor Laboratory Press ISSN1072-0502/99 \$5.00

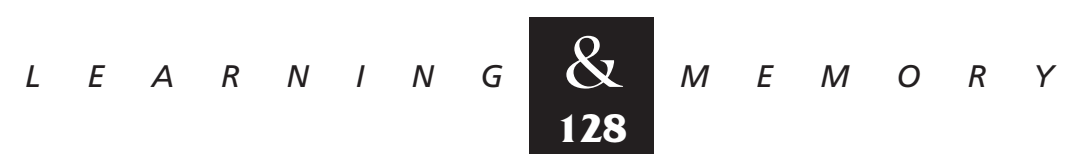


fibers is important for plasticity at the parallel fiber to Purkinje cell spine synapse (Marr 1969; Albus 1971). Although Marr (1969) and Albus (1971) both proposed that plasticity would occur in the form of a change in synaptic efficacy, previous experiments have demonstrated morphological changes in Purkinje cell dendrites and the number of synapses per neuron (Floeter and Greenough 1979; Pysh and Weiss 1979; Greenough et al. 1986; Black et al. 1990). The present study investigated (1) the importance of conjunctive stimulus presentation for Purkinje cell morphological plasticity and (2) whether or not such plasticity is restricted to the spiny branches, targets of parallel fiber synapses.

Eye-blink conditioning has been shown to depend on the two excitatory afferent inputs that are central to Marr's and Albus's models. In eye-blink conditioning, a conditioned stimulus (CS), such as a tone, is paired with an unconditioned stimulus (US), such as an air puff that elicits an eyeblink. After numerous pairings, the tone begins to elicit an eye-blink just before the air puff. The anterior interpositus nucleus and cerebellar cortex receive CS input via mossy fibers from the pontine nuclei and US input via climbing fibers from the inferior olive (for reviews, see Lavond et al. 1993; Anderson and Steinmetz 1994). Neurophysiological activity in the interpositus nucleus and cortical lobule HVI precedes and models the conditioned response (CR). Activity in both areas predicts learning; lesions of both markedly impair conditioned responding. Cerebellar cortical lesions impair CR amplitude and timing (Lavond et al. 1993; for review, see Anderson and Steinmetz 1994).

In the present study, stimulation of the lateral and dorsolateral pontine nuclei (DLPN) was used as a CS to more focally direct CS information to lobules HVI (Larsell's), Crus I and II, and the paramedian lobule (Hoddevik 1975). Pontine stimulation trained animals follow the same conditioning rules as tone or light trained animals (Steinmetz et al. 1986; Steinmetz 1990); similarly, lesions of lobules HVI, Crus I and II, and the paramedian lobule impair CRs to DLPN stimulation CSs (B. Knowlton, G. Beekman, D. Lavord, J. Steinmetz, and R. Thompson, unpubl.).

Given the power of eyeblink conditioning to delineate the effects of conjunctive activation of mossy fibers and climbing fibers (paired CS-US) from those of nonconjunctive activation (unpaired CS-US), we examined its morphological effects on Purkinje cell dendrites. Comparisons with a naive control were used to examine any nondifferential effects of CS and US activation.

Quantification of dendritic length and numbers provides a measure of postsynaptic space. For a constant density of spines on the Purkinje cell spiny branches, a change in the length and number of dendrites should be a good indicator of a change in the number of synapses. In support of the assertion, dendritic length measures in enriched animals have been good predictors of the magnitude of change in the numbers of synapses per neuron later confirmed with electron microscopic analysis (for review, see Greenough 1984). Quantification of dendritic length in Golgi-stained material provides the opportunity to sample throughout the lobule of interest more thoroughly than can feasibly be done with electron microscopy.

\section{Materials and Methods}

\section{SUBJECTS}

Subjects were 15 male New Zealand white rabbits ( 7 paired, 5 unpaired, and 3 naive) weighing between 2.2 and $3 \mathrm{~kg}$. The naive rabbits received no electrode implantation and no training.

Twelve subjects were implanted with monopolar stimulating electrodes in the pontine nucleus. Animals were anesthetized with ketaminerompun $(60 \mathrm{mg} / \mathrm{kg}$ and $6 \mathrm{mg} / \mathrm{kg}$, respectively) followed by halothane (2\%-3\%). Electrodes with exposed tips of 150-250 $\mu \mathrm{m}$ were placed in the pontine nuclei with stereotaxic coordinates of 10.5 $\mathrm{mm}$ posterior to bregma, $2.5 \mathrm{~mm}$ lateral from the midline suture, and $20.5 \mathrm{~mm}$ ventral from bregma with bregma $1.5 \mathrm{~mm}$ above $\lambda$. Single-pulse stimulation at this site produces mossy fiber-evoked potentials in Larsell's HVI lobule of cerebellar cortex (Steinmetz et al. 1985, 1989). Within the paired and unpaired group, approximately the same number of animals had electrodes implanted in the left and right hemispheres. Electrode location was verified histologically. After removing animals with improper electrode placements, the paired group had four animals with left hemisphere implants and three animals with electrodes in the right hemisphere. In the unpaired group, two animals had electrodes in the left hemisphere, and three had electrodes in the right hemisphere. These animals received 1 week of recovery and $1 \mathrm{hr}$ of adaptation to the conditioning apparatus. Three paired animals and one unpaired animal were trained in the laboratory of R. Thompson (USC, Los Angeles).

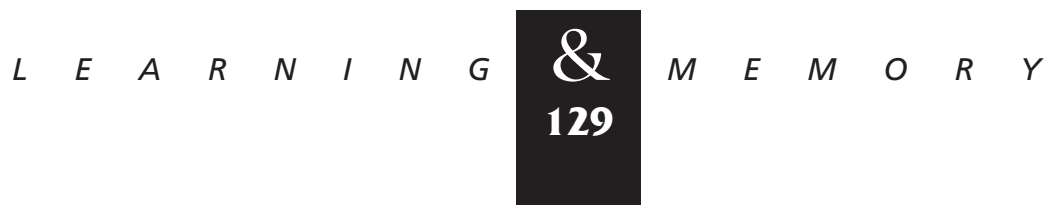


Four paired animals and four unpaired animals were trained in the laboratory of J. Steinmetz (Indiana University, Bloomington). The three naive animals were acquired from the same breeder as the animals trained in the Steinmetz laboratory but were delivered to and sacrificed in the laboratory of W. Greenough (University of Illinois, Urbana). The naive rabbits were not adapted or restrained. Although the delivery of naive rabbits to the Greenough lab is a potential confounding factor in this study, it should be pointed out that within the paired and unpaired groups there are also rabbits from different breeders. Variability among animals from different breeders would be expected to be greater than variability among animals from the same breeder delivered at different times. Tissue processing was kept as similar as possible across all three groups; the same investigator sacrificed all animals except for two paired animals and stained all the tissue. Sectioning, staining, and neuron tracing from the naive animals also overlapped in time with the sectioning, staining, and tracing of paired and unpaired animals obtained from the other labs.

\section{BEHAVIORAL TRAINING}

Animals in the paired group received unilateral stimulation of the pontine nuclei $(200 \mathrm{~Hz}, 350$ msec, 200-250 $\mu \mathrm{A})$, paired with a coterminating air puff (100 $\left.\mathrm{msec}, 2.1 \mathrm{~N} / \mathrm{cm}^{2}\right)$ directed at the cornea contralateral to the electrode. Nictitating membrane movement was measured with a minitorque potentiometer. CRs were operationally defined as greater than or equal to a $0.5-\mathrm{mm}$ movement of the nictitating membrane. All paired animals received 12 blocks of nine trials daily (eight paired trials and one CS alone trial). The mean intertrial interval was $30 \mathrm{sec}$ (range 20-40 sec). Animals in the unpaired group received the same number and type of pontine stimulation and air puff presentations, but the stimuli were presented in a pseudorandom order at random intervals with a range of 1-32 seconds. Both groups received a mean of 12 days (range $=8-14$ ) of training. Animals given paired training all learned to criterion (eight CRs out of nine trials) and received overtraining for a mean of 9 days (range $=7-11$ ). None of the animals in the unpaired group showed evidence of learning, responding at a characteristic spontaneous rate of $1.0 \%$ in CS periods. Approximately $24 \mathrm{hr}$ following the last training session, electrolytic lesions were made to localize the electrode track, and rabbits were anesthetized with sodium pentobarbital and exsanguinated or decapitated.

For analysis, the hemispheres of the paired and unpaired animals were treated separately; the hemisphere ipsilateral to the eye receiving the air puff has been shown by lesion studies to be critical to expression of the CR (Lavond et al. 1984, 1985). Both hemispheres in the naive group were pooled for analysis.

\section{HISTOLOGY AND DATA COLLECTION}

Fresh cerebella were immersed in Golgi-Cox solution (Glaser and Van der Loos 1981). Once staining was complete, as determined from test sections, Larsell's HVI lobule was embedded and sectioned at $120 \mu \mathrm{m}$ parallel to the relatively twodimensional Purkinje cell dendritic plane. The same investigator sacrificed all the animals except for two and stained and sectioned all of the tissue. Slides were coded so that experimenters were blind to individual hemisphere conditions.

Purkinje cells sampled had to lie completely within the plane of section as indicated both by the presence of a cell body and by dendrites extending the full distance to the pia mater. Because the dendritic trees of Purkinje cells are relatively two-dimensional, one rarely, if ever, encounters a truncated branch when the plane of sectioning is parallel to the plane of the dendritic tree. As a result, little information is lost in two-dimensional drawings when sections are cut transverse to the long axis of the folia. In addition to the above constraints, sampled cells had to be completely stained with no indication of discontinuity of stain in any part of the dendritic tree. Typically, these constraints limited the number of cells that could be sampled. Two hundred and fifty-five Purkinje cells were analyzed with a two-step process beginning with camera lucida drawings at a magnification of 706x. Drawings were then digitized with customized dedicated software. The digitizing program was validated by comparison with manual measurements from model and traced neurons with various characteristics (e.g., double and triple bifurcations).

Purkinje cells have large, relatively smooth "main" branches from which small branches densely packed with spines ("spiny" branches) arise. A spiny branch arbor was defined as a cluster of spiny branches that arose from a single point on a main branch. The first order spiny branch was operationally defined as a branch with more than

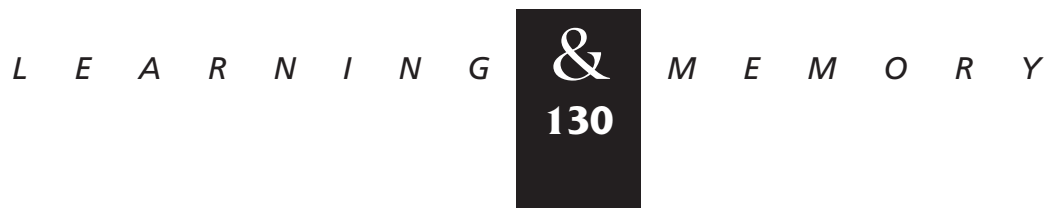


one mushroom-shaped spine and less than half of the diameter of the primary main dendrite. To ensure systematic random sampling, every fifth spiny branch arbor encountered moving centrifugally from the soma was traced with a camera lucida, starting on alternating sides of the Purkinje cells at a random position between one and five. The origin of all spiny branch arbors was indicated on the main branch drawings. If a branch arbor was truncated or too dense to follow every branch, an adjacent spiny branch arbor was drawn, if possible. When both adjacent branches could be drawn, one was selected by coin toss. The entire Purkinje cell main branch arbor was drawn. Main and spiny branch drawings were separately digitized using a data tablet and customized software. Location of the top of the cell body and the pia were entered to allow comparison among the top, middle, and bottom thirds of the molecular layer; spiny branch arbors were categorized according to the third in which their origin occurred. A representative tracing appears in Figure 1. The data were analyzed with the SAS GLM ANOVA.

Inhibitory interneurons were traced with a three-dimensional computer tracking system that encodes three-dimensional information (DeVoogd et al. 1981). No distinction was made between stellate and basket cells, although cells were coded as to whether they were in the upper or lower half of the molecular layer. Sampled cells had complete staining and full dendritic arbors within the section. For the 525 cells drawn, all dendrites were traced. Individual dendrites were subsequently categorized by how many branchpoints occurred between the dendrites and the cell body (branch order) for the purpose of analyzing differences in branches at different orders.

At the time of sacrifice, the brain stem was immersed in sucrose-formalin and subsequently frozen and sectioned. Sections were stained with cresyl violet and Prussian blue and examined to verify electrode placements (see Fig. 2).

\section{Results}

To analyze the effects of the unilateral training (ipsilateral vs. contralateral to air puff) and of the type of training (paired, unpaired, naive), a general linear model analysis of variance (ANOVA) (training group by hemisphere), treating rabbits as independent measures, was performed. The hemispheres in the naive group were assigned to the contralateral hemisphere condition. No interactions were found on any measures. Student-Newman-Keuls post hoc analysis $(\alpha=0.05)$ was performed for measures that were significantly different.

The aggregate length of main branches was $27 \%$ greater in the naive group than in the paired and unpaired groups $[F(2,1)=13.47, P<0.0001$; Fig. 3A]. The naive group had 29\% more spiny branch arbors per Purkinje cell than the paired and unpaired groups $[F(2,1)=23.75, P<0.001$; Fig. 3B]. Mean length of spiny branch arbors was not
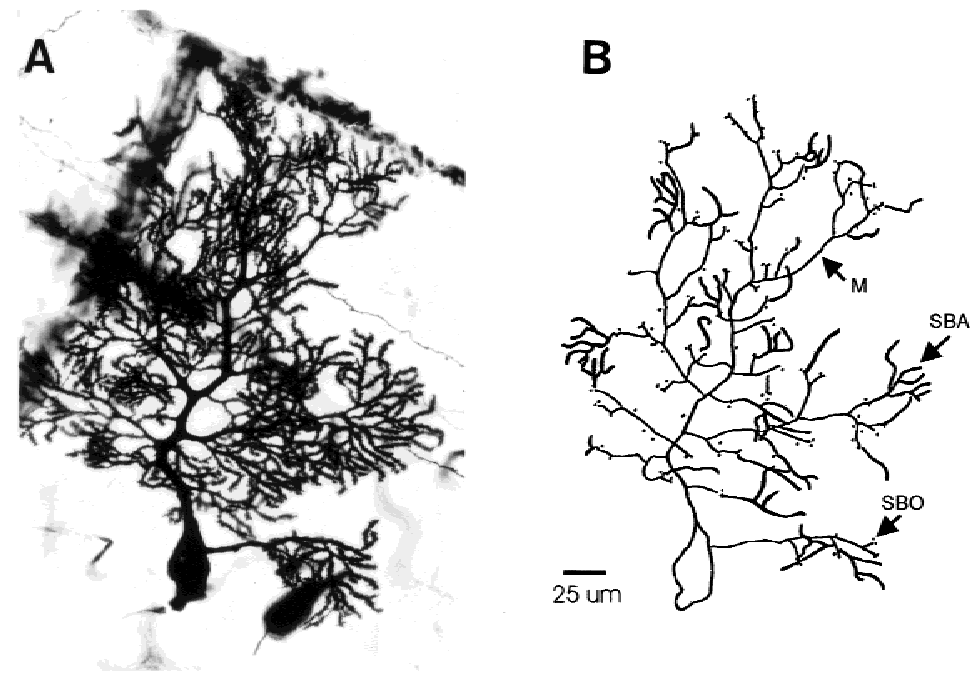

Figure 1: The Golgi-stained Purkinje cell in $A$ is represented by the camera lucida drawing in $B$. (B) Main branches (thin lines; e.g., see M) were encoded separately from spiny branch arbors (thick lines; e.g., see SBA). All spiny branch arbor origins were indicated by dots (e.g., see SBO), but only a sample of spiny branch arbors were drawn entirely. The average length of spiny branch arbors was reported along with the average number of branches per arbor. Individual branch orders on spiny branches were analyzed for number and length. Total spiny branch arbor length per cell was calculated by multiplying the average arbor length by the number of arbors per cell. Discontinuities in the drawing do not represent discontinuities in the cell traced. The relatively two-dimensional dendritic tree of Purkinje cells lies within the $120 \mu \mathrm{m}$ thick sections, thereby providing the opportunity to trace cells with a camera lucida while losing little morphological information. The Golgi-stained Bergmann fiber to the left of the Golgi-stained Purkinje cell in $A$ was below the plane of the dendritic tree and did not interfere with the tracing of dendrites.

$$
\begin{array}{lllllllllllllll} 
& E & A & R & N & I & N & G & \underset{\mathbf{1 3 1}}{\boldsymbol{Z}} & M & E & M & O & R & Y
\end{array}
$$




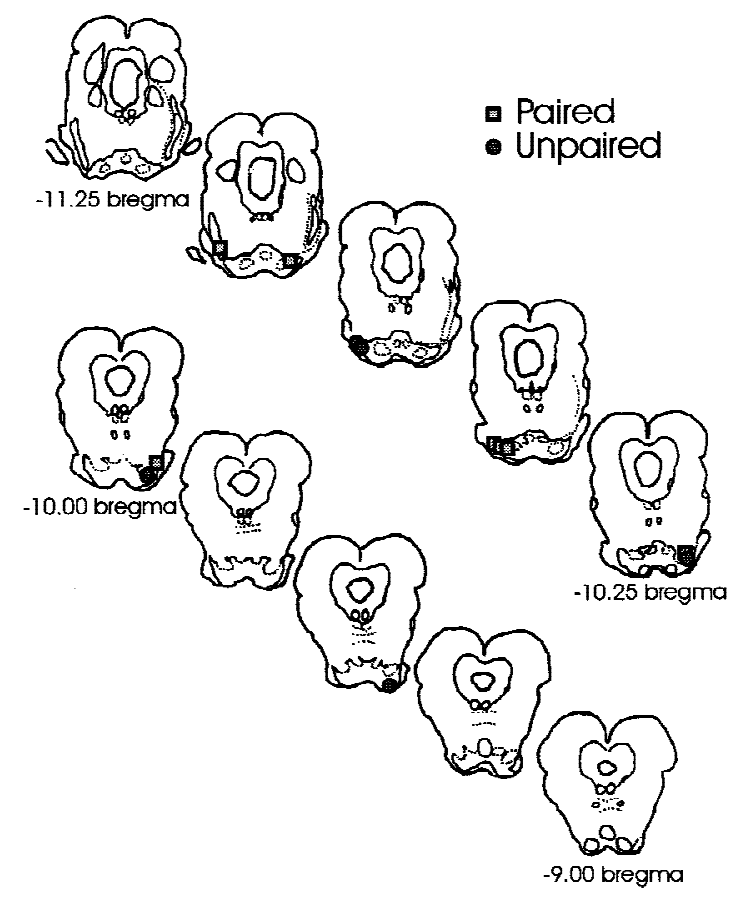

Figure 2: Electrode placements for the animals in this study. Squares represent placements in paired animals, and circles represent placements in unpaired animals. Of the paired animals, four had electrodes on the left side and three had electrodes placed on the right side. In the unpaired group, two had electrode placements on the left side and three had placements on the right side. The left hemisphere is represented on the left side of each brainstem slice. The outlined areas in the ventral portion of each diagram represent the pontine nuclei.

significantly different across groups, but there was a trend toward $18 \%$ larger arbors in the paired and unpaired groups than the naive group $[F(2,1)=3.29, P<0.0546]$. Likewise, the average number of branches per spiny branch arbor did not differ across groups, but the paired and unpaired groups had a trend toward $17 \%$ more branches per arbor than the naive group $[F(2,1)=3.21, P<0.0584]$. To obtain an estimate of the total spiny branch length per cell, the number of spiny branch arbors was multiplied by the average arbor length. On this measure, the naive group was significantly greater $(20 \%)$ than the paired and unpaired groups $[F(2,1)=6.8, P<0.01$; Fig. $3 \mathrm{C}]$. The naive group had significantly greater total dendritic length $(21 \%)$ than the paired and unpaired groups $[F(2,1)=8.2, P<0.01$; Fig. 3D $]$. To be sure that there were no differences between hemispheres ipsilateral and contralateral to the air puff, a general linear model ANOVA, training condition (paired, unpaired) by hemisphere (ipsilat- eral, contralateral), was performed on the paired and unpaired animals only. No significant training or hemisphere main effects or interactions were seen in any measure.

All analyses were subsequently performed separately within each of the outer, middle, and inner thirds of the molecular layer. As neurons become impregnated by the Golgi-Cox procedure, the stain works its way from the cell body to the pial surface and from proximal to distal branches; the outer regions of the Purkinje cells are most likely to exhibit any effects of incomplete impregnation. Effects that are restricted to the outer third of the molecular layer could suggest differential staining rather than morphological plasticity. Inhibitory interneurons were also analyzed across the outer and inner halves of the molecular layer. In the inner half of the molecular layer, basket cells make up the majority of inhibitory interneurons, whereas the stellate cells make up the majority of inhibitory interneurons in the outer half. These analyses aimed to identify differences restricted to the inhibitory interneurons within each half of the molecular layer.

For all measures previously reported as significant, similar trends were noted within each third of the molecular layer. Total dendritic length was significantly higher in the inner third of the molecular layer in the naive group than in the paired group $[F(2,1)=3.63, \quad P<0.05]$. Main branch length was significantly greater in the naive group than in the paired and unpaired group in each third of the molecular layer $[F(2,1)=8.55, P<0.01$; $F(2,1)=3.91, P<0.05, F(2,1)=3.45, P<0.05$; inner, middle, and outer third, respectively]. The number of spiny branch arbors in the naive group was significantly higher in the inner and middle thirds of the molecular layer $[F(2,1)=6.44$, $P<0.01 ; F(2,1)=5.24, P<0.05$; inner and middle third, respectively].

No significant differences in total dendritic length or in the total number of branches for inhibitory interneurons were seen across groups or hemispheres (see Table 1). The naive group had a significantly greater total dendritic length of first order branches $[F(2,1)=7.45, P<0.01$; Table 1]. No differences were seen in the length of branches across groups or hemispheres at other orders. Differences in the number of branches at each order can be seen in Table 1. The unpaired group had a tendency to have fewer branches at the first two orders and a greater number of branches at the fifth through eighth orders. By analyzing group dif-

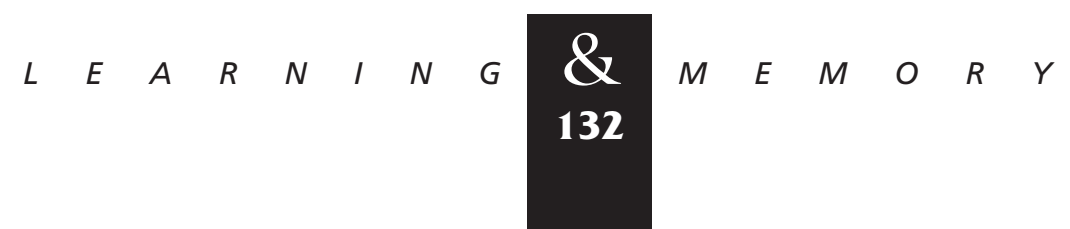



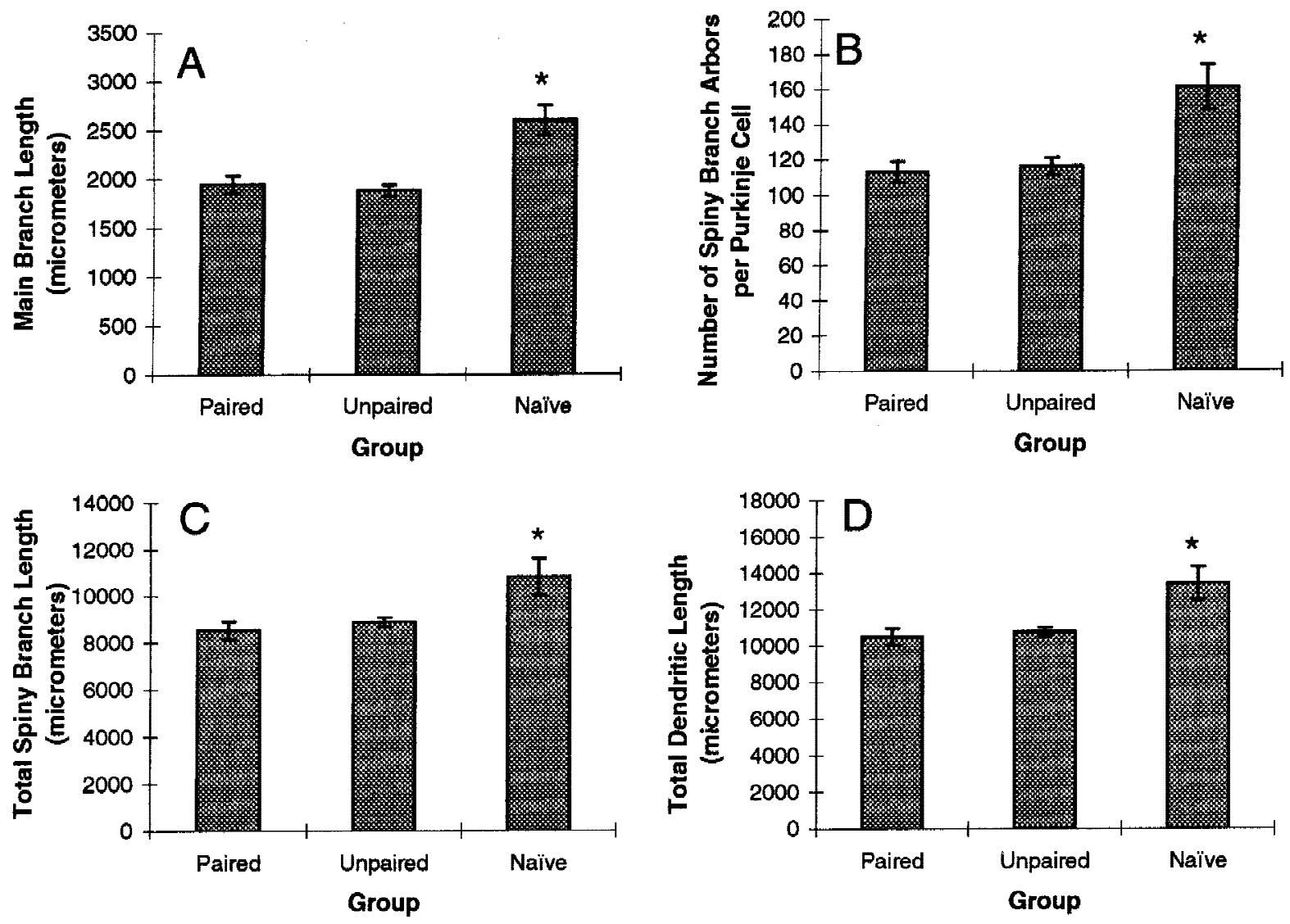

Figure 3: (A) Main branch dendritic length. The length of all main branches added together is greater in the naive group than in the paired and unpaired groups $(P<0.0001)$. Bars represent grand means for each group with S.E.M. $(B)$ Number of spiny branch arbors. The average number of spiny branch arbors per Purkinje cell is greater in the naive group than in the paired and unpaired groups $(P<0.001)$. Bars represent grand means for each training group with S.E.M. $(C)$ Total spiny branch dendritic length. The number of arbors per Purkinje cell was multiplied by the mean spiny branch arbor length to obtain an estimate of the total spiny branch arbor length per cell. The greater arbor numbers in the naive group outweighed the tendency for greater arbor length in the paired and unpaired groups so that the naive group had significantly more total spiny branch dendritic length per cell $(P<0.01)$. (D) Total dendritic length per Purkinje cell. Total dendritic length was estimated by multiplying the number of spiny branch arbor origins by the mean spiny branch arbor length and adding the total length of all main branches $(P<0.01)$.

ferences in inhibitory interneurons in the outer half of the molecular layer and in inhibitory interneurons in the inner half of the molecular layer, we found no evidence that the two inhibitory interneuron samples responded differently to the treatment conditions.

In an analysis of data obtained from an initial group of animals (Anderson et al. 1991), it was found that there were more 5th and 6th order Purkinje cell spiny branches in the unstimulated hemisphere of paired animals than in the stimulated hemisphere. The number of 5 th and 6 th order branches in the paired hemisphere was equal to the numbers found in both hemispheres in the unpaired animals. There was, thus, a decrease in branches in the stimulated hemisphere relative to the unstimulated hemisphere. In the present study, no significant differences were found in the numbers of branches at any order. The present data differs from the data initially reported in that additional animals were added and some of the original animals were removed because the stimulating electrodes were not in the lateral pontine nucleus. Furthermore, some neurons initially studied were deleted here for technical reasons (e.g., incomplete dendritic arbor and stain quality).

\section{Discussion}

Purkinje cell dendritic arbors from both hemi-

$$
\begin{array}{lllllllllllllll}
\hline & E & A & R & N & I & N & G & \underset{133}{\mathbf{Z}} & M & E & M & O & R & Y
\end{array}
$$


Table 1: Inhibitory interneurons

\begin{tabular}{|c|c|c|c|c|c|c|}
\hline \multirow[b]{2}{*}{ Variable/Group } & \multicolumn{2}{|c|}{ Paired $(n=7)$} & \multicolumn{2}{|c|}{ Unpaired $(n=5)$} & \multicolumn{2}{|c|}{ Naive $(n=3)$} \\
\hline & mean & S.E.M. & mean & S.E.M. & mean & S.E.M. \\
\hline \multicolumn{7}{|c|}{ Whole cell data } \\
\hline Total length & 4208.62 & 412.08 & 4173.19 & 283.35 & 4046.92 & 820.62 \\
\hline Total branches & 28.66 & 1.18 & 31.11 & 0.77 & 27.73 & 1.57 \\
\hline \multicolumn{7}{|c|}{ Branch order data } \\
\hline \multicolumn{7}{|c|}{ Number of bifurcating and terminating branches } \\
\hline order 1 & 3.71 & 0.12 & $3.27^{\mathrm{a}}$ & 0.10 & 3.89 & 0.15 \\
\hline order 2 & 6.32 & 0.19 & $5.77^{\mathrm{a}}$ & 0.14 & $6.82^{\mathrm{a}}$ & 0.31 \\
\hline order 3 & 7.66 & 0.27 & 7.71 & 0.15 & 7.81 & 0.42 \\
\hline order 4 & 5.96 & 0.33 & 6.75 & 0.32 & 5.47 & 0.45 \\
\hline order 5 & 3.21 & 0.36 & 4.24 & 0.25 & 2.48 & 0.28 \\
\hline order 6 & 1.28 & 0.19 & 1.95 & 0.19 & 0.84 & 0.17 \\
\hline order 7 & 0.36 & 0.08 & 0.89 & 0.12 & 0.23 & 0.07 \\
\hline \multicolumn{7}{|c|}{ Total length of branches at each order } \\
\hline order 1 & 239.80 & 17.77 & 193.37 & 17.29 & $358.19^{a}$ & 41.01 \\
\hline order 2 & 821.13 & 80.40 & 708.87 & 71.55 & 1028.11 & 204.28 \\
\hline order 3 & 1480.31 & 138.38 & 1433.91 & 126.59 & 1355.29 & 254.69 \\
\hline order 4 & 1048.45 & 141.49 & 1039.68 & 116.06 & 871.25 & 263.30 \\
\hline order 5 & 439.81 & 90.17 & 509.67 & 80.86 & 311.64 & 82.84 \\
\hline order 6 & 142.35 & 46.34 & 177.59 & 25.00 & 88.37 & 27.26 \\
\hline order 7 & 25.83 & 10.11 & 75.53 & 6.08 & 18.71 & 5.15 \\
\hline
\end{tabular}

$\mathrm{a}(P<0.01)$ In the case that two groups differed but the third did not, the two groups that differ both have a symbol.

spheres of the paired and unpaired groups were smaller than those of naive rabbits. No differences were observed between the paired group and unpaired group or between hemispheres ipsilateral and contralateral to the air puff within these groups. Thus, morphological change did not appear to be limited to conjunctive stimulus presentations or to the hemisphere that, according to lesion-based data, is most critical to performance. These reported changes are not apparently related to conditions that correspond to the production of the associatively CR.

Purkinje cells from the paired and unpaired groups had less total dendritic length and less main branch length than Purkinje cells from naive rabbits. Trained rabbits also had fewer spiny branch arbors per Purkinje cell than naive rabbits. Whereas there was a trend for spiny branch arbor size to be larger in the paired and unpaired groups than in the naive group, trained rabbits had less total spiny branch arbor length per Purkinje cell than naive rabbits. As a whole, the data indicate activation-induced reorganization of the Purkinje cell dendritic tree by retraction of main branch dendrites and a decrease in the number of spiny branch arbors. That conclusion is consistent with the activation-induced increase (via $20 \mu \mathrm{A}$ square pulses at $50 \mathrm{~Hz}$ ) in motor cortical synaptic density reported by Keller et al. (1992) and the changes in dendritic and axonal arborizations in cats receiving unpaired as well as paired presentations of cortical stimulation (0.4-1.0 mA) and foot shock (Rutledge et al. 1974). The difference in the direction of the changes may reflect the differences in the regions investigated (cerebrum vs. cerebellum).

The group differences in proximal as well as distal branches mitigates the possibility that the group effects represent a difference in the quality of the stain. Staining with Golgi-Cox begins in the soma and gradually impregnates more distal dendrites. Purkinje cell dendrites in the bottom third of the molecular layer are the first to stain and, hence, should exhibit the least differences if the results reflect differential staining across groups. In contrast, we see the largest effects in the bottom third of the molecular layer that contains proximal dendrites.

The CS in this study involved the injection of 200-250 $\mu \mathrm{A}$ of current to the lateral pontine nuclei. This current could have damaged pontine 
cells immediately adjacent to the electrode tip. We cannot assess potential damage because marking lesions were used to verify the stimulation site. Learning rates, however, were not slower as would be predicted if the results were owing to damage. The percentage difference in total dendritic length reported here is within the range of differences found in the cerebellum after behavioral treatment (Greenough et al. 1986; Black et al. 1990). Furthermore, main branches, which are not targets of the mossy fiber-parallel fiber pathway, also differed across groups. We observed no signs of damage such as the giant spines reported by Hillman and Chen (1984) following parallel fiber transection. The stellate cells are also primarily innervated by the mossy fiber-parallel fiber pathway, yet the stimulated groups did not differ in total dendritic length or total number of branches from the naive group. Rutledge et al. (1974) used higher current for a stimulation CS and looked at dendrites immediately postsynaptic to the stimulated cells. The length and number of dendrites on cells contacted by the stimulated cells increased, whereas we found a decrease in cells two synapses away from the site of stimulation.

Stress associated with surgery is unlikely to have caused the decrease in dendrites because stress-associated dendritic atrophy in highly susceptible structures, such as CA3 of the hippocampus, are only known to occur with chronic stress or glucocorticoid administration (McEwen 1994). We have no way of knowing whether Purkinje cell dendritic atrophy might result from damage related to surgery. It is interesting to note, however, that the paired animals learned at the same rate as animals with noninvasive training methods. The fact that stellate cell dendritic trees, which receive the same parallel and climbing fiber afferent excitatory input from the pons, did not differ across groups, along with results for individual spiny branch length (which tended to be larger in the operated groups) further suggests that there is no generalized damage resulting from surgery.

The changes in Purkinje cell morphology in the unpaired rabbits are consistent with data from Gould and Steinmetz (1996). Over 5 days of unpaired training, various sites in lobule HVI exhibited increases in US-related unit activity. Following paired training, most of these sites exhibited wellsculpted CR-related activity. However, sites that had a decrease in US-related activity over 5 days of unpaired training did not develop the CR-related activity that typically developed during subsequent paired training trials. These data show that several forms of plasticity occur during unpaired training trials; the form taken predicts how the cells will respond to subsequent paired training.

The dendritic changes were not limited to the hemisphere corresponding to the eye receiving the air puff. This may be owing to nearly equal bilateral projections of the pontine nuclei (Mihailoff 1983; Payne 1983). Likewise, the trigeminal nucleus probably projects air puff information bilaterally to the cerebellum (VanHam and Yeo 1992), and more data are needed regarding the inferior olivary projections. B.E. Polenchar and M.M. Patterson (unpubl.) have reported tone and air puff evoked neural responses and CR-related neural activity bilaterally in the dentate/interpositus nucleus. It is yet unclear whether their results are owing to bilateral projections from precerebellar nuclei or intracerebellar projections. The bilateral nature of the effects reported are consistent with bilateral cerebellar activation in humans undergoing eye-blink conditioning (Blaxton et al. 1996) and bilateral cerebellar PKC- $\gamma$ immunoreactivity in rabbits (E.A. Van der Zee, I.F. Palm, M.A. Kronforest, E.T. Maizels, M. Shanmugam, M. Hunzicker-Dunn, and J.F. Disterhoft, unpubl.).

Modelers have hypothesized that the climbing fiber may be a "teacher" or carrier of error-related input because climbing fibers have an all-or-none impact on the Purkinje cell, and they fire at a relatively low frequency (Marr 1969; Albus 1971). In that hypothesized role, the modulatory influence of the climbing fibers causes an increase (or decrease) in the number of connections or a strengthening (or weakening) of existing synapses between parallel fibers and Purkinje cell spiny branches. The possibility of plasticity at other Purkinje cell synaptic sites has usually been ignored. However, because the amount of postsynaptic material for both parallel and climbing fiber synapses changed in the present experiment, the results suggest that both types of excitatory synapses onto the Purkinje cell (climbing fiber and parallel fiber to Purkinje synapses) may exhibit plasticity. These findings are not completely surprising in light of several findings. Rossi et al. (1991) used 3-acetyl pyradine (3-AP) to produce partial lesions of the inferior olive in adult rats. The remaining climbing fibers sprouted and reinnervated nearby Purkinje cells. Greenough et al. (1986) found that old rats exhibited altered main branch organization that could not be explained by age-related regression. These reports emphasize the need for future em-

$$
\begin{array}{llllllllllllllll} 
& E & A & R & N & I & N & G & \boldsymbol{Q} \\
135 & M & E & M & O & R & Y
\end{array}
$$


pirical work aimed at investigating the possibility that main branches and their corresponding afferents are capable of structural plasticity as a form of adaptation.

The mechanisms responsible for the development and maintenance of the CR do not appear to be the morphological changes investigated here. The morphology of other areas (or morphological features not investigated here) could be changing. For example, cells in the interpositus nucleus or the numbers of synaptic contacts in the cerebellar cortex or deep nuclei could be changing. Alternatively, the CR may not require detectable morphological change. For example, synaptic efficacy changes could involve alterations at the level of receptors or ion channels resulting in a change in the efficacy of existing synapses (e.g., long-term depression). Because we cannot rule out other possible forms of learning, the present findings do not address the issue of whether or not the cerebellar cortex is the site necessary for learning the CR in this paradigm.

Preliminary results (B. Anderson, K. Relucio, C. Mohr, C. Logan, B. Knowlton, F. Davis, A. Hawkins, J. Thompson, J. Steinmetz, R. Thompson, and W. Greenough, unpubl.) did suggest an associative effect on Purkinje cell dendritic morphology, namely a decrease in Purkinje neuron spiny branches in the stimulated hemisphere relative to the unstimulated hemisphere, consistent with a mechanism like long-term depression (Ito 1984). Although excluded here, our initial report included some animals with electrode placements that were not in the pontine nuclei but that learned the associative task. In eyeblink conditioning, regardless of where an electrical brain CS is delivered, the cerebellum is always necessary for learning and memory (Solomon et al. 1986; Steinmetz et al. 1986; Knowlton et al. 1988; Knowlton and Thompson 1989, 1992). If those animals had cerebella that were weakly activated by polysynaptic pathways rather than by direct mossy fiber stimulation, then the direct stimulation of the mossy fiber pathway in the present groups could have overridden more subtle effects of the polysynaptic activation. This possibility leaves us wary of generalizing the results to the standard training protocol of a peripheral tone paired with an air puff. In the more standard protocol, the tone is likely to activate a more complex circuit leading to an unknown effect on the cerebellum.

The present data indicate that temporally contiguous stimuli have no greater ability to induce
Purkinje cell dendritic structural change than nonpaired stimulus presentations. Too many differences exist between the naive group and the paired and unpaired groups to indicate what specific components of the training experience are related to the differences reported here. Rabbits in both the paired and unpaired groups learned something about their training experience. The present data, however, are particularly relevant to associative learning, those forms of learning that are dependent on temporal or contingent relationships. The present results taken with those of Keller et al. (1992) and Rutledge et al. (1974) suggest that structural plasticity depends on mere activation and is not limited to associative conditions. Activation-induced effects could underlie the nonassociative forms of learning and memory, which have been much harder to define. However, the fact that activation, or use, could have long-term effects on neuronal morphology is interesting in itself, regardless of its relationship to learning per se.

\section{Acknowledgments}

We thank David Krupa, Rodney Swain, and A.J. Annala for help in animal training. We are grateful to Franz Davis, Alex Hawkins, and Steven Lee who helped draw neurons for this experiment or pilot versions of this experiment. Nick Kissebeth developed the digitizing program and maintained the three-dimensional computer tracing system. Rodney Swain provided helpful discussion and comments on this manuscript. This study was supported by grant $\mathrm{MH} 40631$. Stipend support was provided by training grants PHS HD-07333 and MH18412.

The publication costs of this article were defrayed in part by payment of page charges. This article must therefore be hereby marked "advertisement" in accordance with 18 USC section 1734 solely to indicate this fact.

\section{References}

Albus, J.S. 1971 A theory of cerebellar function. Math. Biosci. 10: 25-61.

Anderson, B.J. and J.E. Steinmetz. 1994. Cerebellar and brainstem circuits involved in classical eyeblink conditioning. Rev. Neurosci. 5: 251-273.

Black, J.E., K.R. Isaacs, B.J. Anderson, A.A. Alcantara, and W.T. Greenough. 1990. Learning causes synaptogenesis, whereas motor activity causes angiogenesis, in cerebellar cortex of adult rats. Proc. Natl. Acad. Sci. 87: 5568-5572.

Blaxton, T.A., T.A. Zeffiro, J.D.E. Gabriele, S.Y. Bookheimer, M.C. Carrillo, W.H. Theodore, and J.F. Disterhoft. 1996. Functional mapping of human learning: A positron emission tomography activation study of eyeblink conditioning. J. Neurosci. 16: 4032-4040.

DeVoogd, T. J., F.-L. Chang, M.K. Floeter, M.J. Jencius, and W.T. Greenough. 1981. Distortions induced in neuronal

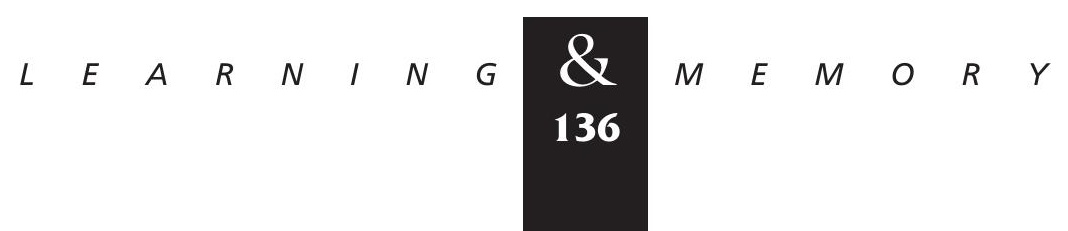


quantification by camera lucida analysis: Comparisons using a semiautomated data acquisition system. J. Neurosci. Meth. 3: $285-294$.

Floeter, M.K. and W.T. Greenough. 1979. Cerebellar Plasticity: Modification of Purkinje cell structure by differential rearing in monkeys. Science 206: 227-229.

Glaser, E.M. and H. Van der Loos. 1981. Analysis of thick brain sections by observe-reverse computer microscopy: Applications of a new high clarity Golgi-Nissl stain. J. Neurosci. Meth. 4: 117-125.

Gould, T.J. and J.E. Steinmetz. 1996. Changes in rabbit cerebellar cortical and interpositus nucleus activity during acquisition, extinction, and backward classical eyelid conditioning. Neurobiol. Learn. Mem. 65:17-34.

Greenough, W.T. 1984. Structural correlates of information storage in the mammalian brain: A review and hypothesis. Trends Neurosci. 7: 229-233.

Greenough, W.T., J.W. McDonald, R.M. Parnisari, and J.E. Camel. 1986. Environmental conditions modulate degeneration and new dendritic growth in cerebellum of senescent rats. Brain Res. 380: 136-143.

Hillman, D.E. and S. Chen. 1984. Reciprocal relationship between size of postsynaptic densities and their number: Constancy in contact area. Brain Res. 295: 325-343.

Hoddevik, G.H. 1975. The pontocerebellar projection onto the paramedian lobule in the cat: An experimental study with the use of horseradish peroxidase as a tracer. Brain Res. 95: 291-307.

Ito, M. 1984. The cerebellum and neural control. Raven Press, New York, NY.

Keller, A., K. Arissian, and H. Asanuma. 1992. Synaptic proliferation in the motor cortex of adult cats after long-term thalamic stimulation. J. Neurophys. 68: 295-308.

Knowlton, B. and R.F. Thompson. 1989. Stimulation of the lateral septum acts as a much more effective CS than stimulation of the medial septum in classically conditioned eyeblink response. Behav. Neurosci. 103: 206-208.

1992. Conditioning using a cerebral cortical CS is dependent on the cerebellum and brainstem circuitry. Behav. Neurosci. 106: 509-517.

Knowlton, B.J., D.G. Lavond, and R.F. Thompson. 1988. The effects of lesions of cerebellar cortex on retention of the classically conditioned eyeblink response when stimulation of the lateral reticular nucleus is used as the conditioned stimulus. Behav. Neural Biol. 49: 293-301.

Lavond, D.G., D.A. McCormick, and R.F. Thompson. 1984. A nonrecoverable learning deficit. Physiol. Psych. 12: 103-110.

Lavond, D.G., T.L. Hembree, and R.F. Thompson. 1985. Effect of kainic acid lesions of the cerebellar interpositus nucleus on eyelid conditioning in the rabbit. Brain Res. 326: $179-182$.
Lavond, D.G., J.J. Kim, and R.F. Thompson. 1993. Mammalian brain substrates of aversive classical conditioning. Annu. Rev. Psychol. 44: 317-342.

Marr, D. 1969. A theory of cerebellar cortex. J. Physiol. 202: $437-470$.

McEwen, B.S. 1994. The plasticity of the hippocampus is the reason for its vulnerability. Semin. Neurosci. 6: 239-246.

Mihailoff, G.A. 1983. Intra- and interhemispheric collateral branching in the rat pontocerebellar system, a fluorescence double-label study. Neurosci. 10: 141-160.

Payne, J.N. 1983. Axonal branching in the projections from precerebellar nuclei to the lobulus simplex of the rat's cerebellum investigated by retrograde fluorescent double labeling. J. Comp. Neurol. 213: 233-240.

Pysh, J.J. and G.M. Weiss. 1979. Exercise during development induces an increase in Purkinje cell dendritic tree size. Science 206: 230-231.

Rossi, F., L. Wiklund, J.J.L. Van der Want, and P. Strata. 1991. Reinnervation of cerebellar Purkinje cells by climbing fibres surviving a subtotal lesion of the inferior olive in the adult rat. I. Development of new collateral branches and terminal plexuses. J. Comp. Neurol. 308: 513-535.

Rutledge, L.T., C. Wright, and J. Duncan. 1974. Morphological changes in pyramidal cells of mammalian neocortex associated with increased use. Exper. Neurol. 44: 209-228.

Solomon, P.R., J.L. Lewis, J. LoTurco, J.E. Steinmetz, and R.F. Thompson. 1986. The role of the middle cerebellar peduncle in acquisition and retention of the rabbit's classically conditioned nictitating membrane response. Bull. Psychon. Soc. 24: 65-78.

Steinmetz, J.E. 1990. Classical nictitating membrane conditioning in rabbits with varying interstimulus intervals and direct activation of cerebellar mossy fibers as the CS. Behav. Brain Res. 38: 97-108.

Steinmetz, J.E., D.G. Lavond, and R.F. Thompson. 1985. Classical conditioning of the rabbit eyelid response with mossy fiber stimulation as the conditioned stimulus. Bull. Psychon. Soc. 23: 245-248.

1989. Classical conditioning in rabbits using pontine nucleus stimulation as a conditioned stimulus and inferior olive stimulation as an unconditioned stimulus. Synapse 3: 225-233.

Steinmetz, J.E., D.J. Rosen, P.F. Chapman, D.G. Lavond, and R.F. Thompson. 1986. Classical conditioning of the rabbit eyelid response with a mossy fiber stimulation CS: I. Pontine nuclei and middle cerebellar peduncle stimulation. Behav. Neurosci. 100: 878-887.

VanHam, J.J. and C.H. Yeo. 1992. Somatosensory trigeminal projections to the inferior olive, cerebellum and other precerebellar nuclei in rabbits. Eur. J. Neurosci. 4: 302-317.

Received June 26, 1998; accepted in revised form March 1, 1999.

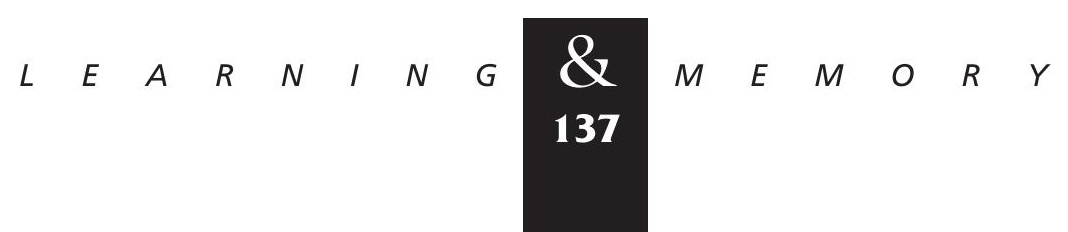




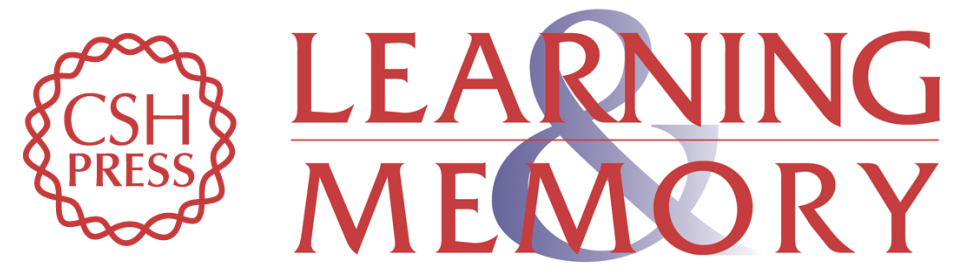

\section{Effects of Paired and Unpaired Eye-Blink Conditioning on Purkinje} Cell Morphology

Brenda J. Anderson, Karen Relucio, Karl Haglund, et al.

Learn. Mem. 1999, 6:

Access the most recent version at doi:10.1101//m.6.2.128

References This article cites 28 articles, 4 of which can be accessed free at: http://learnmem.cshlp.org/content/6/2/128.full.html\#ref-list-1

License

Email Alerting Receive free email alerts when new articles cite this article - sign up in the box at the Service top right corner of the article or click here. 